\title{
Faire bouger un peu les lignes
}

Entretien avec Laurent Le Bon

David Cascaro et Laurent Le Bon

\section{(2) OpenEdition}

Journals

Édition électronique

URL : https://journals.openedition.org/rbnu/2725

DOI : $10.4000 /$ rbnu. 2725

ISSN : 2679-6104

Éditeur

Bibliothèque nationale et universitaire de Strasbourg

Édition imprimée

Date de publication : 1 novembre 2012

Pagination : 62-67

ISSN : 2109-2761

Référence électronique

David Cascaro et Laurent Le Bon, "Faire bouger un peu les lignes », La Revue de la BNU [En ligne], 6 | 2012, mis en ligne le 01 novembre 2012, consulté le 08 août 2021. URL : http:// journals.openedition.org/rbnu/2725; DOI : https://doi.org/10.4000/rbnu.2725 Attribution - Pas d'Utilisation Commerciale - Partage dans les Mêmes Conditions 4.0 International. 


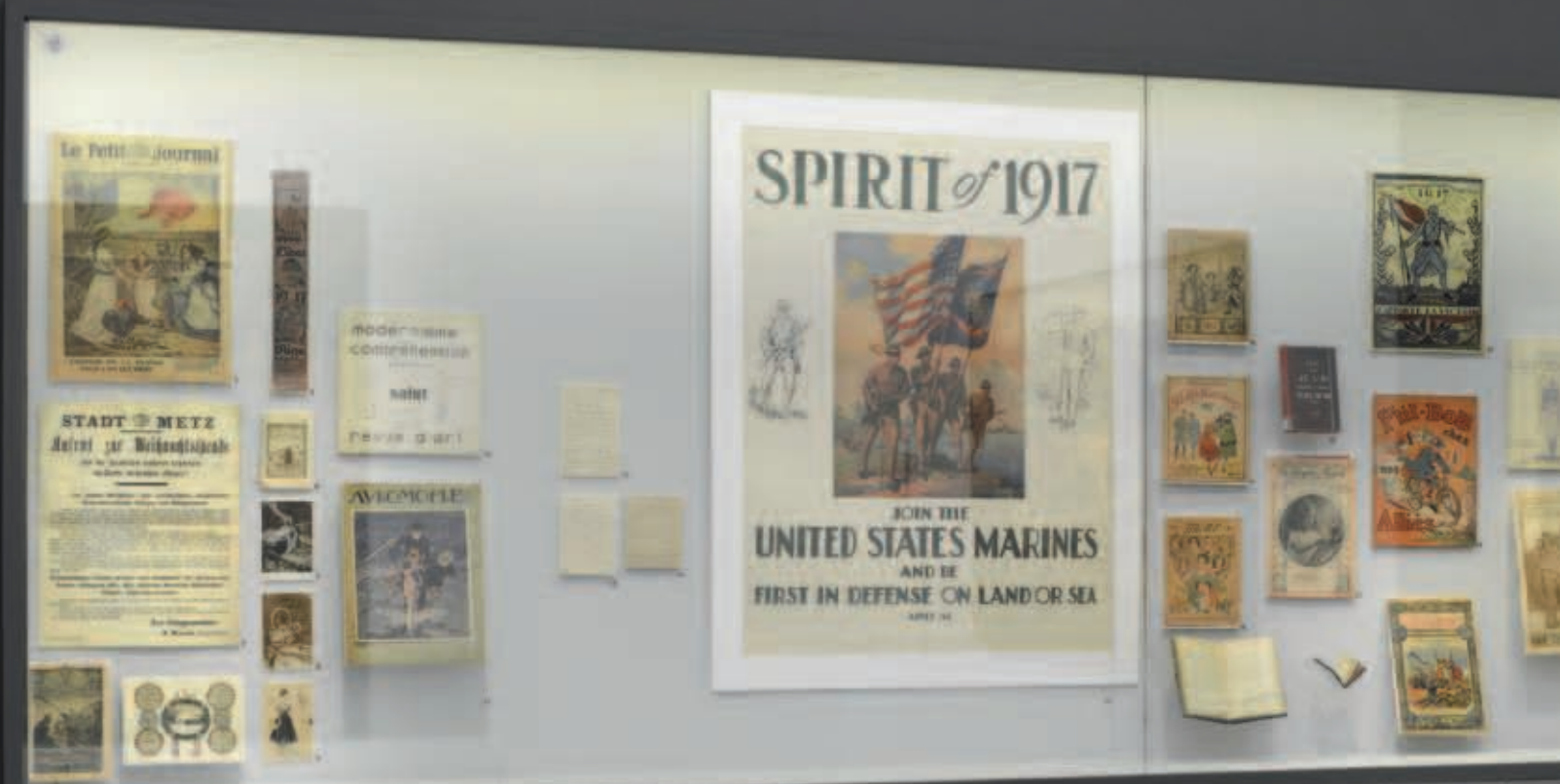

(19) 


\section{FAIRE BOUGER UN PEU LES LIGNES :}

\section{entretien avec Laurent Le Bon}

Laurent Le Bon est l'actuel directeur du Centre Pompidou-Metz. C'est sous sa direction que celui-ci a été inauguré, avec l'exposition Chefs-d'œuvre ? qui a attiré en Lorraine plusieurs centaines de milliers de visiteurs. Il a aussi, avec Claire Garnier, été à l'origine de l'exposition 1917 qui vient de fermer ses portes. Il tente ici d'expliquer sa démarche de commissaire, en réfléchissant sur la fonction pédagogique de l'exposition et du catalogue, et en montrant comment ceux-ci peuvent, par la présentation des savoirs, aider à mieux faire comprendre ces derniers. Il s'entretient ici avec David Cascaro, directeur de la Haute école des arts du Rhin qui dispense à Strasbourg un enseignement reconnu dans les domaines de la communication graphique et de la scénographie.

\section{Quelle place occupe le catalogue dans la production de vos expositions ? Quel soin portez-vous à son élaboration ? Quel rôle revient aux graphistes et typographes d'une telle entreprise?}

De manière traditionnelle, les catalogues complètent les propos développés au sein des expositions. Le Centre Pompidou-Metz a la chance de pouvoir éditer, le plus souvent seul, ses propres catalogues, ce qui nous donne une liberté précieuse et rare pour publier des ouvrages assez ambitieux. Le catalogue étant voué à pérenniser un propos d'exposition temporaire, il mérite qu'on lui apporte un soin maximal, avec une contrainte de planning - le catalogue doit paraitre dans la grande majorité des cas lors de l'ouverture de l'exposition. Les graphistes accompagnent les directeurs d'ouvrages souvent commissaires d'expositions - pour apporter une réponse graphique et formelle à une proposition de concept et de contenu (sommaire puis chemin de fer ${ }^{1}$ ). En cela, leur rôle s'apparente à celui du scénographe pour une exposition : concevoir un écrin pour un corpus d'œuvres ou de textes. 

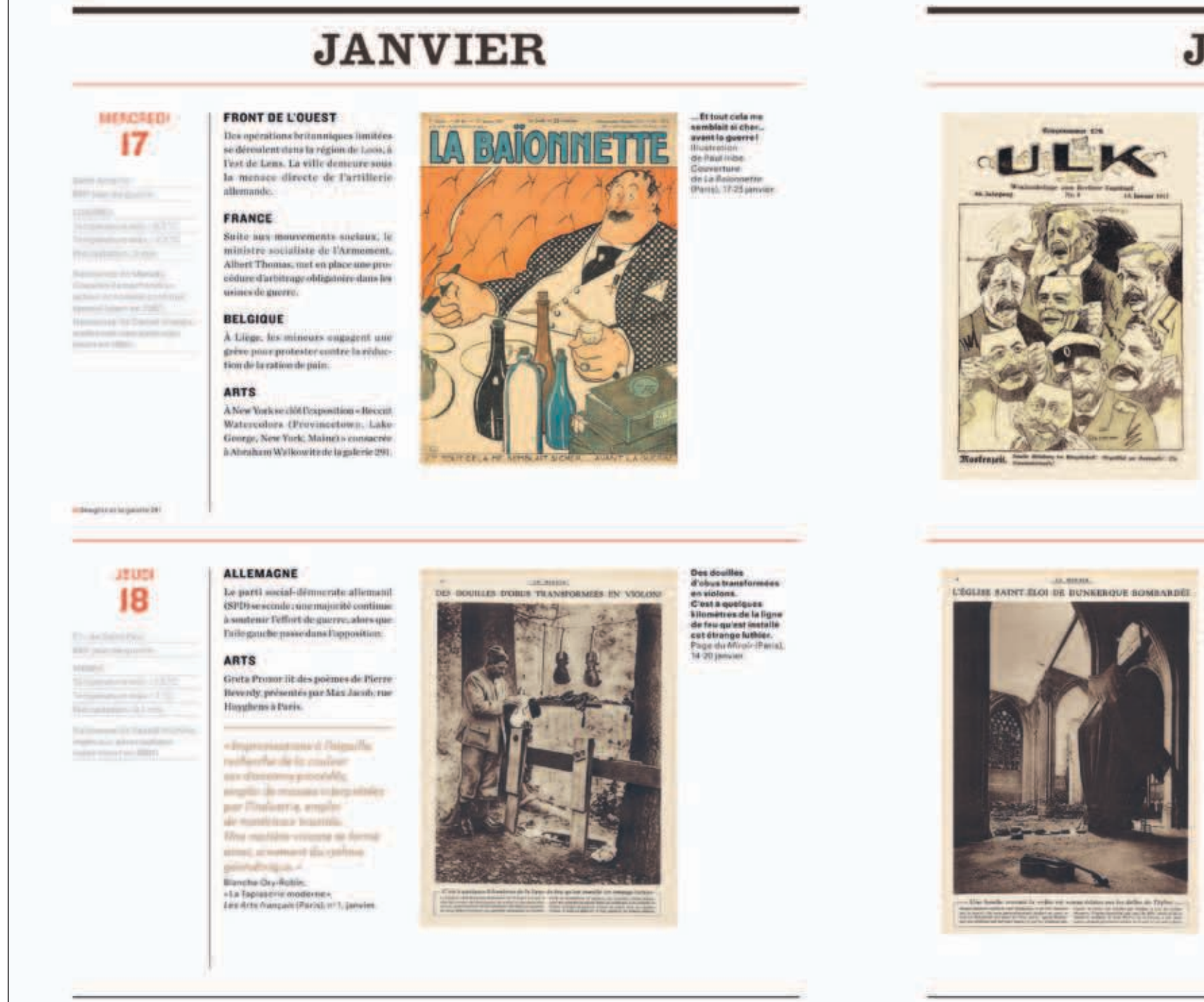

\section{JANV}
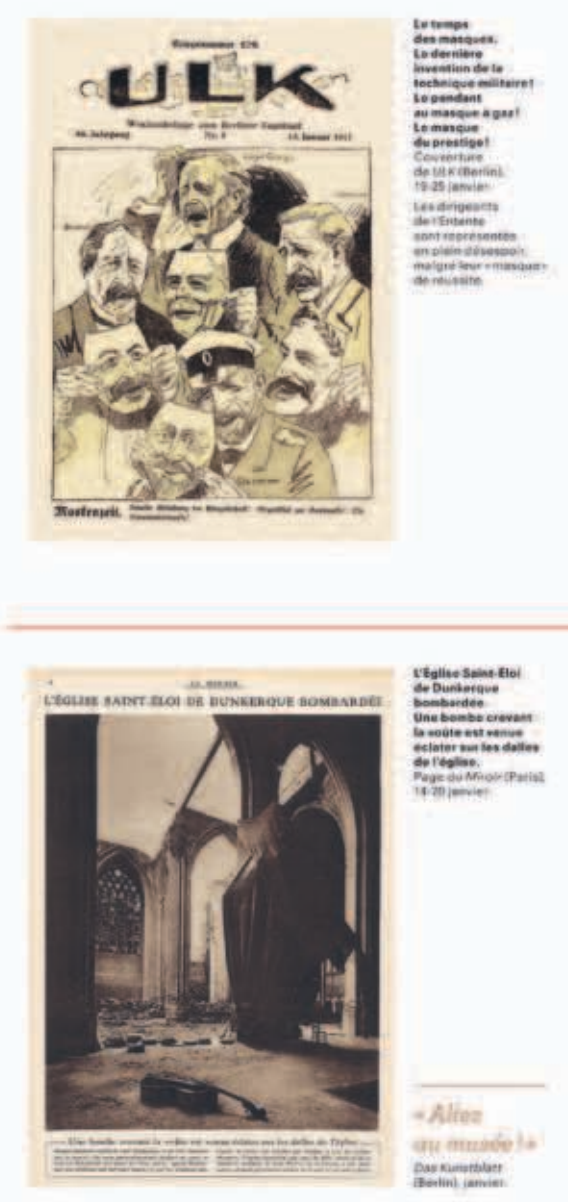

Chacun des catalogues que vous avez produits est construit autour d'idées originales qui se révèlent à la lecture des pages : ordre alphabétique à la manière d'un index de l'ensemble des chapitres pour Dada (plaçant par exemple le sommaire à la lettre S), ordre chronologique de l'entrée des œuvres dans les collections du Musée national d'art moderne pour Chefs-d'auvre ?, etc. Quelle est l'approche retenue pour le catalogue de l'exposition 1917?

Nous avons conçu un ouvrage qui propose une approche du sujet complémentaire de celle de l'exposition. En effet, tandis que le parcours de l'exposition est entièrement thématisé, les trois parties du catalogue déclinent successivement une série d'essais généraux, un dictionnaire et un journal de l'année. Dans un premier temps, sept essais apportent des éclairages généraux sur l'année 1917. Le deuxième volet de l'ouvrage est pensé comme un dictionnaire de l'année 1917, avec environ 225 notices courtes, thématiques ou biographiques, consacrées aux hommes, événements, lieux, disciplines, problématiques culturelles, artistiques ou quotidiennes qui ont " fait " cette année 1917. Cette section est illustrée d'une grande partie des œuvres et documents présentés dans l'exposition. Le dernier volet se présente comme un journal de l'année, 
1917, catalogue sous la direction de Claire Garnier et Laurent Le Bon, conception graphique Aurore Jannin et Laurent Pinon, Editions du Centre Pompidou-Metz, 2012.

Vue d'une double page de la section " 1917 de janvier à décembre ". l'édition papier, car il permet de diffuser des contenus différents. Nous avons expérimenté cela à l'occasion de l'exposition Ronan et Erwan Bouroullec. Bivouac : la présence de tablettes numériques dans l'exposition a été complétée par l'édition d'un catalogue traditionnel. C'est sans doute le grand défi qui nous attend de pouvoir offrir à la fois des livres de qualité sur support papier qui auront toujours au moins un public de niche et des supports numériques ambitieux.

Comment envisagez-vous personnellement l'acte de lire ? Peut-il être comparé aux déplacements du corps des visiteurs dans un espace d'exposition ? Jean-Max Colard développe actuellement une recherche fascinante sur la " littérature exposée ", qui évoque ces interactions entre littérature et expositions. Cette « littérature exposée " est ainsi un monde en soi aux ramifications multiples et aux modalités de lectures très diverses. La lecture est présente dans presque toutes les expositions (présence de textes historiques, d'écrits d'artistes ou de textes pédagogiques par exemple). Il existe bien sûr des expositions ayant pour thème la littérature ; dans 1917 comme dans Dada, nous avons essayé de donner à lire au sein d'une exposition d'art et d'histoire. Comme pour l'acte de lecture traditionnel, on peut dans une exposition butiner, papillonner, serpenter, se concentrer...

\section{La plupart des expositions artistiques que vous} avez organisées donnent une part importante, en vis-à-vis des œuvres, à la documentation (livres, revues, photographies d'accrochages), autrefois remisée dans les marges ou les allées secondaires des musées. Ce dialogue entre objet, artefact et œuvre vous caractérise-t-il ?

La pluridisciplinarité est une caractéristique souvent revendiquée dans les expositions, mais qui est relativement difficile à mettre en pratique, notamment du fait des contraintes de présentation liées à certaines œuvres et aux documents par exemple. Dans l'exposition 1917, nous avons repris le principe que j'avais expérimenté avec Dada, qui consiste à présenter, dans la mesure du possible, les documents dans des vitrines verticales afin de respecter une égalité de traitement et un dialogue permanent avec les autres œuvres. Dans 1917, on a désigné comme " couloir documentaire " l'ensemble des documents ainsi présentés, mais grâce au travail du scénographe Didier Blin, les vitrines constituent en fait le plus souvent le quatrième mur de 


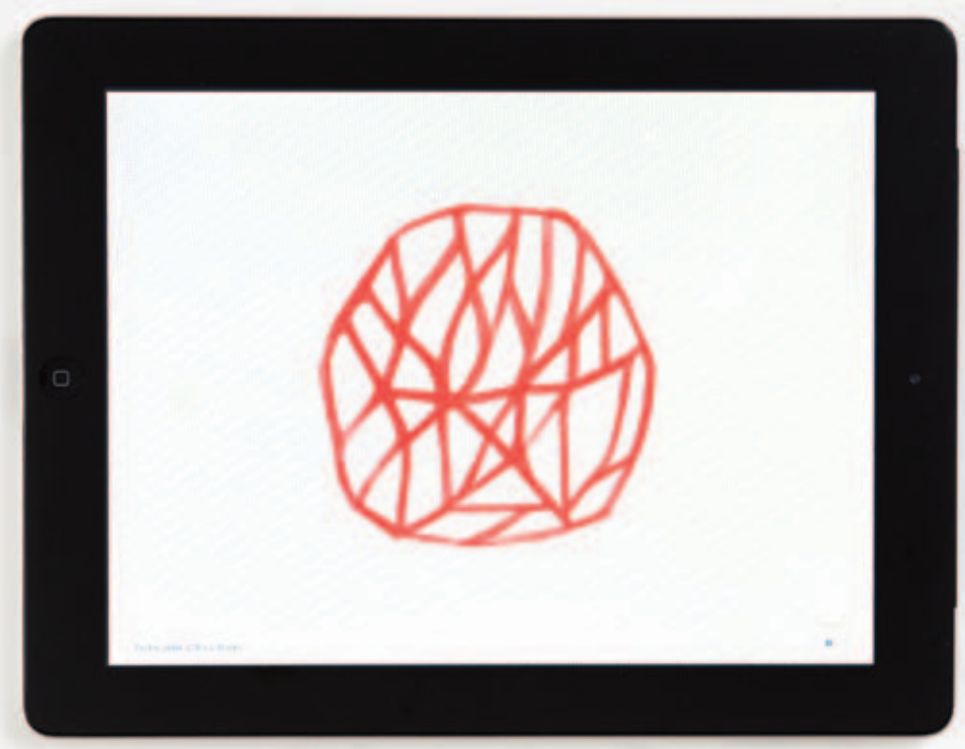

Studio Bouroullec, Cercles, application iPad présentée dans l'exposition

Ronan et Erwan Bouroullec. Bivouac

au Centre Pompidou-Metz

du 7 octobre 2011 au 30 juillet 2012.

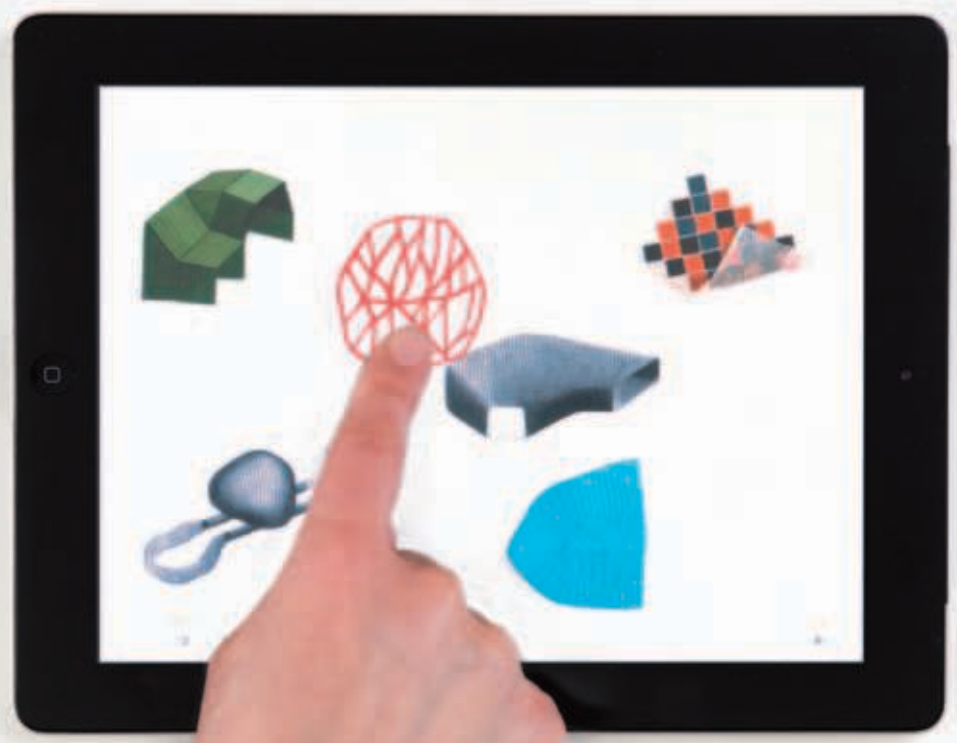


la salle, avec un vis-à-vis qui nous paraissait essentiel. Pour des sujets comme Dada ou 1917, qui concernent des périodes ou des mouvements qui remettaient en cause la hiérarchie des genres traditionnelle, ce parti-pris nous est apparu comme une évidence pour montrer la folie de la création, cette diversité, cette masse de productions artistiques et documentaires accompagnée d'une remise en cause des typologies habituelles des créateurs et des disciplines.

\section{Vous sentez-vous de ce fait l'héritier du musée du Trocadéro et du " white cube " ${ }^{2}$ ?}

Beaucoup de musées, d'historiens d'art, de commissaires, de regardeurs... m'ont influencé. Je ne saurais aisément privilégier l'un ou l'autre. J'essaie en tout cas dans mes expositions de me rapprocher du métier de metteur en scène : on écrit un scénario qu'on essaie de mettre en espace. J'ai évidemment une dette immense envers les grandes expositions du Centre Pompidou (de Paris).

\section{Est-ce que le Centre Pompidou-Metz offre un contexte favorable à cette approche ?}

Je considère que le Centre Pompidou-Metz est un centre d'art et d'essai, un laboratoire qui doit nous permettre de développer des projets, et notamment des expositions, qui ne pourraient pas être faits ailleurs. C'est ce qui m'a incité à proposer l'exposition 1917 qui relève d'un genre atypique. Le Centre Pompidou-Metz bénéficie d'une grande liberté de programmation, qui nous permet de nous inscrire dans des itinérances internationales ou de concevoir nos propres projets, essentiellement monographiques et thématiques. Le Centre Pompidou-Metz a à cœur de faire revivre la quintessence originelle du Centre Pompidou, avec sa pluridisciplinarité et son ambition dans la programmation.

\section{Avec un parti pris très radical - ne présenter} que des œuvres produites la même année l'exposition 1917 réunit un ensemble très dense d'œuvres et de documents d'époque. Que souhaitez-vous déclencher chez le spectateur, tiraillé entre l'expérience esthétique et le désir de connaissance?

Comme souvent, cette exposition vise à apporter connaissance et plaisir esthétique. Avec plus de 1500 œuvres et documents répartis sur environ $2300 \mathrm{~m}^{2}$, nous avons souhaité montrer la folie de la guerre et la folie créatrice de cette année 1917, les enchevêtrements, les dialogues, les rencontres, les " concomitances énigmatiques ", pour reprendre l'expression de Jean-Jacques Lebel. Le contenu de l'exposition est effectivement très dense, mais nous constatons, après quelques semaines d'ouverture, que c'est finalement l'émotion qui prime chez la majorité des visiteurs. Devant cette masse, le visiteur ressent la fragilité et la force des individus, et je crois que c'est le plus important.

Vous avez consacré un livre intelligent et sensible au château de Courances (situé en Ile-de-France) ${ }^{3}$, conçu comme un lieu de mémoire autant qu'un lieu de vie. Envisagez-vous autrement l'exposition ou le catalogue, véritables théâtres du monde ?

Cet ouvrage visait à raconter l'histoire de ce château et de son parc et à montrer que ce domaine exceptionnel n'était pas figé dans une époque, qu'il avait évolué au fil des siècles et des diverses influences architecturales. Lorsqu'on se lance dans l'aventure folle et dévoreuse de temps d'un catalogue et d'une exposition (les deux sont le plus souvent intrinsèquement liés), on ne peut pas le faire sans une ambition déraisonnable de faire bouger un peu les lignes et d'amener les spectateurs/ lecteurs (je pense aussi à l'ouvrage d'Harald Szeemann Ecrire les expositions) vers d'autres mondes que ceux d'un quotidien parfois un rien plombant. C'est la magie de nos métiers que de donner corps à ces utopies où l'original a toute sa force et qui me semblent encore dégager plus de force en ces temps de crise et de folie du virtuel.

\section{Propos recueillis par David Cascaro}

